\title{
新型含取代异噁唑结构的吡唑酰胺类衍生物的合成及其生物活性研究
}

\author{
戴 红 ${ }^{a}$ 姚 炜 ${ }^{a}$ 叶林玉 $^{a}$ 方 源 ${ }^{b}$ 石玉军*,a,b \\ 宋 婵 ${ }^{a}$ 李春建 ${ }^{c}$ 石 健 $*, c$ \\ ( ${ }^{a}$ 南通大学化学化工学院 南通 226019) \\ ( ${ }^{b}$ 江苏科技大学环境与化学工程学院 镇江 212003) \\ ( ${ }^{c}$ 南通大学分析测试中心 南通 226019)
}

\begin{abstract}
摘要 为了从吡唑类化合物中寻找具有新的活性物质, 利用亚结构拼接原理, 设计合成了一系列新型含取代异噁唑结 构的吡唑酰胺类衍生物. 利用 ${ }^{1} \mathrm{H} \mathrm{NMR} 、{ }^{13} \mathrm{C} N \mathrm{NR}$ 和元素分析对标题化合物的结构进行了表征. 初步的生物活性测试结 果表明, 部分目标化合物具有较好的杀虫活性. 在测试浓度为 $500 \mu \mathrm{g} / \mathrm{mL}$ 时, 部分化合物对粘虫的防效为 $60 \% \sim 100 \%$, 对蜴虫的杀死率分别为 $50 \% \sim 100 \%$. 另外, 部分化合物对 $\mathrm{HepG}_{2}$ 细胞显示出较好的抗肿瘤活性, 其 $\mathrm{IC}_{50}$ 值为 $14.2 \sim$ $18.9 \mu \mathrm{mol} / \mathrm{L}$.
\end{abstract}

关键词＼cjkstart异噁唑; 吡唑; 酰胺; 合成; 生物活性

\section{Synthesis and Biological Activities of Novel Pyrazole Amide Derivatives Containing Substituted Isoxazole Group}

\author{
Dai, Hong $^{a} \quad$ Yao, Wei $^{a} \quad$ Ye, Linyu $^{a} \quad$ Fang, Yuan $^{b} \quad$ Shi, Yujun ${ }^{*, a, b}$ \\ Song, Chan ${ }^{a} \quad$ Li, Chunjian ${ }^{c} \quad$ Shi, Jian ${ }^{*, c}$ \\ ( ${ }^{a}$ College of Chemistry and Chemical Engineering, Nantong University, Nantong 226019) \\ ( ${ }^{b}$ School of Environmental and Chemical Engineering, Jiangsu University of Science and Technology, Zhenjiang 212003) \\ ( ${ }^{c}$ Analysis and Testing Center, Nantong University, Nantong 226019)
}

\begin{abstract}
In order to find novel pyrazole compounds possessing potent biological activities, a series of novel pyrazole amide derivatives bearing substituted isoxazole moiety were designed and synthesized according to the method of active substructure combination. Their structures were confirmed by ${ }^{1} \mathrm{H}$ NMR, ${ }^{13} \mathrm{C}$ NMR and elemental analysis. The bioassay data indicated that some title compounds had good insecticidal activities. At the concentration of $500 \mu \mathrm{g} / \mathrm{mL}$, some compounds showed $60 \% \sim$ $100 \%$ insecticidal activity against Oriental armyworm, and 50\% 100\% insecticidal activity against Aphis medicaginis. Additionally, some compounds exhibited good anti-cancer activity against HepG2 cells with the $\mathrm{IC}_{50}$ values of $14.2 \sim 18.9 \mu \mathrm{mol} / \mathrm{L}$. Keywords isoxazole; pyrazole; amide; synthesis; biological activity
\end{abstract}

杂环化合物是当前新药发展的主流，而在杂环化合 物中则以含氮杂环为主. 吡唑类化合物作为一类重要的 含氮杂环, 以其优良的杀虫 ${ }^{[1 \sim 4]}$ 、杀菌 ${ }^{[5,6]}$ 、抗病毒 ${ }^{[7]}$ 及 抗肿瘤 ${ }^{[8,9]}$ 等活性而引起药物化学家极大的研究兴趣. 目前, 多种具有广谱生物活性的吡唑类衍生物被相继成 功开发出来, 如日本三菱化学公司研制的杀螨剂吡螨胺
(Tebufenpyrad，图 1)，该化合物具有高效、低毒、持效 期长等特点, 可用于防治多种螨虫 ${ }^{[10]}$, 美国杜邦公司研 究开发的超高效杀虫剂氯虫苯甲酰胺 (Chlorantraniliprole, 图 1)对鳞翅目类害虫如粘虫、小菜蛾等表现出良 好的杀灭效果 ${ }^{[11]}$. 环氧合酶-2 选择抑制剂塞来考昔、镇 痛消炎药安乃近等在人类医疗保健方面也有较为广泛

* Corresponding authors. E-mail: yjshi2015@163.com, gaohbhe2015@aliyun.com Received February 19, 2017; revised March 9, 2017; published online April 1, 2017.

Project supported by the National Natural Science Foundation of China (No. 21372135), the Research Foundation of the Six People Peak of Jiangsu Province (No. 2013-SWYY-013), the Science and Technology Project Fund of Nantong City (No. MS22015020), and the Postgraduate Research and Practice Innovation Program of Jiangsu Province (No. SJCX17_0640).

国家自然科学基金(No. 21372135)、江苏省 “六大人才高峰” (No. 2013-SWYY-013)、南通市科技计划(No. MS22015020)和江苏省研究生实践创新计 划(No. SJCX17_0640)资助项目. 
的应用 ${ }^{[12,13]}$. 另外, 异噁唑环亦为含氮杂环体系中的重 要一员, 异啞唑类化合物在农药、医药领域发挥着重要

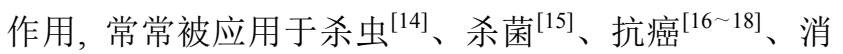
炎 ${ }^{[19]}$ 等方面. 如 $\mathrm{Lu}$ 等 ${ }^{[20]}$ 报道的含氮杂双环 [3.2.1]辛烷 结构的异噁唑啉类化合物 $\mathbf{A}$ 对南方根接线虫表现出良 好的抑制活性(图 1), 在测试剂量为 $20 \mathrm{mg} / \mathrm{L}$ 时, 化合物 A 对南方根接线虫的抑制率为 $100 \%$, Shi 等 ${ }^{[21]}$ 合成的含 氧基丙烯酸酯骨架结构的异啞唑类衍生物 B 对人肝癌 (HepG2)细胞株呈现出优异的抗肿瘤活性(图 1), 其 $\mathrm{IC}_{50}$ 值为 $3.2 \mu \mathrm{mol} / \mathrm{L}$. 此外, 酰胺键是有机化学和药物化学 中较为常见的官能团, 其广泛存在于天然产物和合成药 物中. 酰胺结构单元常作为重要的药效基团引入到母体 化合物分子中, 以改善其理化性质与生物活性 ${ }^{[22 ~ 27]}$. 为 了进一步从吡唑类化合物中寻找与发现高活性化合物, 本研究以吡螨胺为先导化合物, 采用生物电子等排方 法, 选用异噁唑杂环单元代替吡螨胺分子中的苯环, 同 时对其吡唑环 3-位加以修饰, 设计并制备了一系列未见 文献报道的新型含取代异噁唑环结构的吡唑酰胺类化 合物(图 2). 通过 ${ }^{1} \mathrm{H} N \mathrm{NMR},{ }^{13} \mathrm{C} \mathrm{NMR}$ 和元素分析等手段 对目标化合物的结构进行了表征. 初步的生物活性测试 结果表明, 某些目标化合物表现出较好的杀虫作用, 部 分标题化合物显示出良好的抗肿瘤活性. 其合成路线如 Scheme 1 所示.

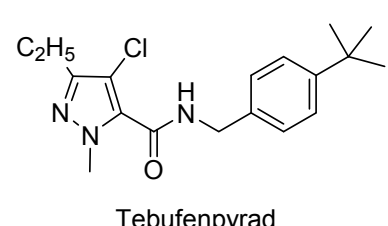

Tebufenpyrad<smiles>CN1C2CC(=NOCC3CC(c4ccc([N+](=O)[O-])cc4)=NO3)CC1C2</smiles><smiles>CCOC(=O)C(C#N)=C(NCc1cc(-c2ccc(F)cc2)on1)SC</smiles>

图 1 吡螨胺、氯虫苯甲酰胺、化合物 $\mathbf{A}$ 和 $\mathbf{B}$ 的化学结构式 Figure 1 Chemical structures of tebufenpyrad, chlorantraniliprole, compounds $\mathbf{A}$ and $\mathbf{B}$

\section{1 结果与讨论}

\section{1 目标化合物的合成}

目标化合物 10 是通过 1-甲基-3-取代基-4-氯吡唑-5甲酰氯(5)与 5-芳基异噁唑-3-甲基胺(9)的缩合反应制备 而得. 以化合物 $10 f$ 为研究对象, 探讨了不同的反应条

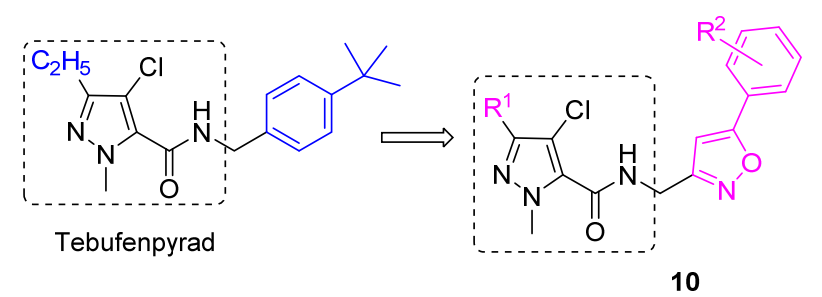

图 2 目标化合物 $\mathbf{1 0}$ 的分子设计示意图

Figure 2 Design strategy of the title compounds 10

件对化合物 $10 \mathrm{f}$ 收率的影响, 其相关数据列于表 1 中. 从表 1 可发现, 采用三乙胺作缚酸剂, 四氢呋喃(THF) 为溶剂, 室温反应 $8 \mathrm{~h}$, 是制备目标化合物的较佳方法, 化合物 $10 \mathrm{f}$ 的收率为 $58 \%$, 该方法反应条件温和，后处 理简单. 因此, 采用该法顺利地制备了其它的目标物.

表 1 不同反应条件对目标化合物 $10 f$ 收率的影响

Table 1 Effects of reaction conditions on the synthesis of the target compound $\mathbf{1 0 f}$

\begin{tabular}{ccllc}
\hline Entry & Base & Solvent & \multicolumn{1}{c}{ Reaction condition } & Yield/\% \\
\hline 1 & Pyridine & $\mathrm{CH}_{2} \mathrm{Cl}_{2}$ & Reflux for $8 \mathrm{~h}$ & 12 \\
2 & Pyridine & $\mathrm{CH}_{3} \mathrm{CN}$ & Reflux for $8 \mathrm{~h}$ & 23 \\
3 & Pyridine & $\mathrm{THF}$ & Reflux for 8 h & 18 \\
4 & $\mathrm{Et}_{3} \mathrm{~N}$ & $\mathrm{CH}_{2} \mathrm{Cl}_{2}$ & Reflux for $8 \mathrm{~h}$ & 45 \\
5 & $\mathrm{Et}_{3} \mathrm{~N}$ & $\mathrm{CH}_{3} \mathrm{CN}$ & Reflux for 8 h & 20 \\
6 & $\mathrm{Et}_{3} \mathrm{~N}$ & $\mathrm{THF}$ & Room temperature for $8 \mathrm{~h}$ & 58 \\
\hline
\end{tabular}

\section{2 化合物的图谱分析}

以目标化合物 $10 \mathrm{a}$ 的核磁氢谱与碳谱数据为例进行 解析. $\delta$ 在 7.71 处的双重峰为苯环上两个氢的吸收峰; $\delta$ 7.33 处的单峰为酰胺基上氢的吸收峰; $\delta 6.97$ 处的双重 峰为苯环上两个氢的吸收峰; $\delta 6.44$ 处的单峰为异噁唑 环上一个氢的吸收峰; $\delta 4.74$ 处的双重峰为与异噁唑环 相连的亚甲基上两个氢的吸收峰; $\delta 4.16$ 处的单峰为吡 唑环 1-位甲基上三个氢的吸收峰; $\delta 3.86$ 处的单峰为苯 环 4-位甲氧基上三个氢的吸收峰; $\delta 2.65$ 处的四重峰为 吡唑环 3-乙基上亚甲基两个氢的吸收峰; $\delta 1.24$ 处的三 重峰为吡唑环 3-乙基上甲基三个氢的吸收峰; $\delta 55.4$ 处 的峰为与苯环 4-位甲氧基碳原子的信号峰; $\delta 40.8$ 处的 峰为与异惡唑环相连的亚甲基碳原子的信号峰; $\delta 35.6$ 处的峰对应于吡唑环 1-位甲基碳原子的信号峰; $\delta 19.2$ 处的峰对应于吡唑环 3-乙基上亚甲基碳原子的信号峰; $\delta 12.8$ 处的峰为吡唑环 3-乙基上甲基碳原子的信号峰.

\section{3 化合物的杀虫活性}

目标化合物 10a $\sim 10$ p 对粘虫(Oriental armyworm)、 蚜虫(Aphis medicaginis)、朱砂叶螨(Tetranychus cinnabarinus)和褐飞虫(Nilaparvata lugens)的杀虫活性测试 结果表明, 所有目标物对朱砂叶螨和褐飞呈均未表现出 杀灭活性, 部分目标化合物对粘虫和蚜虫显示出一定的 

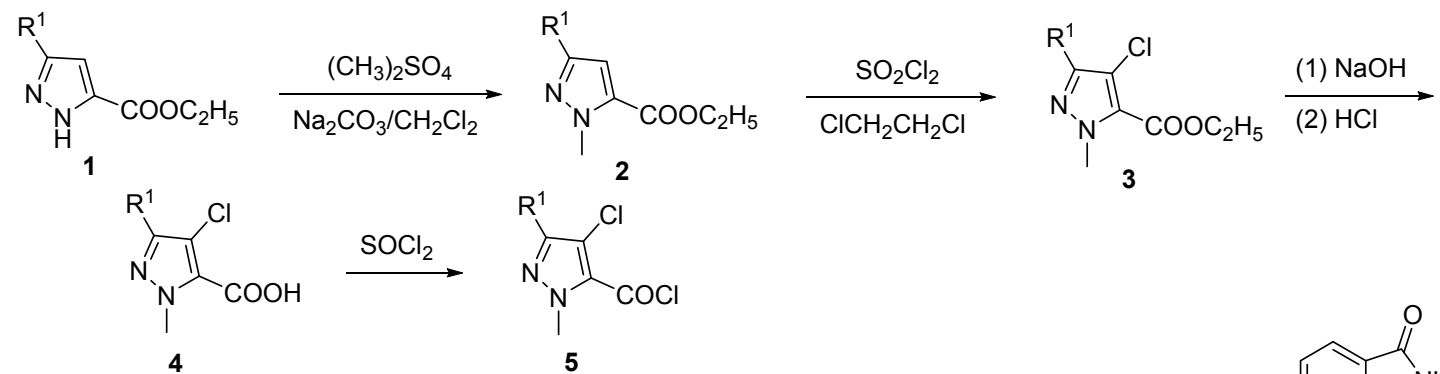<smiles>[R]c1ccc(-c2cc(C(=O)OC)no2)cc1</smiles><smiles>[R][R]1ccc(-c2cc(CO)no2)cc1</smiles>

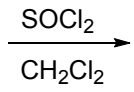

7

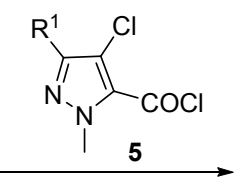

$\mathrm{Et}_{3} \mathrm{~N}, \mathrm{THF}$<smiles>[R2]c1ccc(-c2cc(CCl)no2)cc1</smiles>
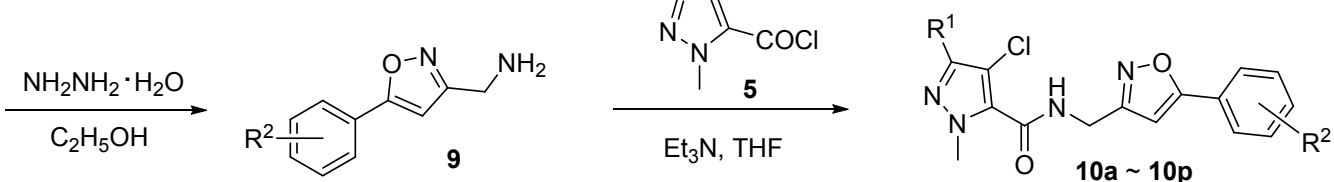

10a: $R^{1}=\mathrm{C}_{2} \mathrm{H}_{5}, \mathrm{R}^{2}=4-\mathrm{OCH}_{3} ; 10 b: \mathrm{R}^{1}=\mathrm{C}_{2} \mathrm{H}_{5}, \mathrm{R}^{2}=4-\mathrm{CH}_{3} ; 10 \mathrm{c}: \mathrm{R}^{1}=\mathrm{C}_{2} \mathrm{H}_{5}, \mathrm{R}^{2}=\mathrm{H} ; 10 \mathrm{~d}: \mathrm{R}^{1}=\mathrm{C}_{2} \mathrm{H}_{5}, \mathrm{R}^{2}=3-\mathrm{F} ; 10 \mathrm{e}: \mathrm{R}^{1}=\mathrm{C}_{2} \mathrm{H}_{5}$, $R^{2}=4-F ; 10 f: R^{1}=C_{2} H_{5}, R^{2}=4-C l ; 10 g: R^{1}=C_{2} H_{5}, R^{2}=3-B r ; 10 h: R^{1}=C_{2} H_{5}, R^{2}=3,4-\left(O_{3} H_{3}\right)_{2} ; 10 i: R^{1}=C_{2} H_{5}, R^{2}=2,4-F_{2}$; 10j: $\mathrm{R}^{1}=\mathrm{C}_{2} \mathrm{H}_{5}, \mathrm{R}^{2}=3,4-\mathrm{Cl}_{2} ; 10 \mathrm{k}: \mathrm{R}^{1}=4-\mathrm{CH}_{3} \mathrm{OC}_{6} \mathrm{H}_{4}, \mathrm{R}^{2}=4-\mathrm{F} ; 10 \mathrm{l}: \mathrm{R}^{1}=3-\mathrm{FC}_{6} \mathrm{H}_{4}, \mathrm{R}^{2}=4-\mathrm{Cl} ; 10 \mathrm{~m}: \mathrm{R}^{1}=4-\mathrm{FC}_{6} \mathrm{H}_{4}, \mathrm{R}^{2}=4-\mathrm{Cl}$; 10n: $\mathrm{R}^{1}=4-\mathrm{ClC}_{6} \mathrm{H}_{4}, \mathrm{R}^{2}=4-\mathrm{Cl} ; 10 \mathrm{o}: \mathrm{R}^{1}=4-\mathrm{IC}_{6} \mathrm{H}_{4}, \mathrm{R}^{2}=4-\mathrm{Cl} ; 10 \mathrm{p}: \mathrm{R}^{1}=2,4-\mathrm{F}_{2} \mathrm{C}_{6} \mathrm{H}_{3}, \mathrm{R}^{2}=4-\mathrm{F}$

图式 1 目标化合物 $\mathbf{1 0}$ 的合成路线

Scheme 1 Synthetic route of the target compounds 10

杀虫活性. 在测试浓度为 $500 \mu \mathrm{g} / \mathrm{mL}$ 时, 化合物 $\mathbf{1 0 k}$,

101, 10n 和 10o 对粘虫的抑制率分别为 $90 \%, 60 \%, 100 \%$ 和 $100 \%$, 其中化合物 10k, 10n 和 10o 对粘虫的杀灭效 果与对照药吡虫啉 $(100 \%)$ 接近; 当测试剂量降至 100 $\mu \mathrm{g} / \mathrm{mL}$ 时, 化合物 10k, 10n 和 100 对粘虫均未表现出杀 虫活性; 此外, 在测试浓度为 $500 \mu \mathrm{g} / \mathrm{mL}$ 时, 化合物 10a， 10b 和 10j 对蚜虫的杀死率分别为 $100 \%, 90 \%$ 和 50\%, 其中化合物 10a 和 10b 对蚜虫的防效与对照药吡 虫啉 $(100 \%)$ 接近; 当测试剂量降至 $100 \mu \mathrm{g} / \mathrm{mL}$ 时, 化合 物 10a 和 $10 \mathrm{~b}$ 对蚜虫均未显示出杀虫效果. 这为今后继 续从事吡唑酰胺类化合物的分子设计合成与生物活性 研究提供了重要的结构选择模式和实验数据.

\section{4 化合物的抗肿瘤活性}

目标化合物 $10 a \sim 10 p$ 对人胃癌(SGC7901)和人肝 癌(HepG2)细胞株的体外抗肿瘤活性数据列于表 2. 从 表 2 可以看出, 目标化合物对 SGC7901 癌细胞均未显示 出明显的抑制活性, 但某些目标化合物对 HepG2 癌细 胞呈现出较好的抑制作用. 当 $\mathrm{R}^{1}=\mathrm{C}_{2} \mathrm{H}_{5}, \mathrm{R}^{2}=4-\mathrm{CH}_{3}, \mathrm{H}$ 或 4-F 时, 化合物 10b, 10c 和 10e 对 HepG2 细胞的抑制 效果相对较好, 其 $\mathrm{IC}_{50}$ 值分别为 $14.2,18.9$ 和 16.4 $\mu \mathrm{mol} / \mathrm{L}$, 要高于或接近于对照药 Sorafenib 的活性 $\left(\mathrm{IC}_{50}=16.2 \mu \mathrm{mol} / \mathrm{L}\right)$. 目前该类型化合物的结构衍生与 抗肿瘤作用研究正在进行中.
表 2 目标化合物 10a 10p 的体外抗肿瘤活性

Table 2 Cytotoxicity of title compounds $10 a \sim 10$ p

\begin{tabular}{ccc}
\hline \multirow{2}{*}{ Compd. } & \multicolumn{2}{c}{$\mathrm{IC}_{50} /\left(\mu \mathrm{mol} \cdot \mathrm{L}^{-1}\right)$} \\
\cline { 2 - 3 } $\mathbf{1 0 a}$ & $\mathrm{SGC} 7901$ & HepG2 \\
$\mathbf{1 0 b}$ & $>20$ & $>20$ \\
$\mathbf{1 0 c}$ & $>20$ & 14.2 \\
$\mathbf{1 0 d}$ & $>20$ & 18.9 \\
$\mathbf{1 0} \mathbf{c}$ & $>20$ & $>20$ \\
$\mathbf{1 0 f}$ & $>20$ & 16.4 \\
$\mathbf{1 0 g}$ & $>20$ & $>20$ \\
$\mathbf{1 0 h}$ & $>20$ & $>20$ \\
$\mathbf{1 0 i}$ & $>20$ & $>20$ \\
$\mathbf{1 0 j}$ & $>20$ & $>20$ \\
$\mathbf{1 0 k}$ & $>20$ & $>20$ \\
$\mathbf{1 0 l}$ & $>20$ & $>20$ \\
$\mathbf{1 0 m}$ & $>20$ & $>20$ \\
$\mathbf{1 0} \mathbf{n}$ & $>20$ & $>20$ \\
$\mathbf{1 0 0}$ & $>20$ & $>20$ \\
$\mathbf{1 0 p}$ & $>20$ & $>20$ \\
Sorafenib & 12.1 & 16.2 \\
\hline
\end{tabular}

\section{2 结论}

本文利用活性基团拼接方法，设计并合成了 16 个 新型含取代异噁唑结构的吡唑酰胺类衍生物. 初步的生 物活性测试结果表明, 部分目标化合物具有较好的杀虫 效果. 在测试浓度为 $500 \mu \mathrm{g} / \mathrm{mL}$ 时，化合物 10k, 101, 10n 
和 100 对粘虫的杀灭效果分别为 $90 \%, 60 \%, 100 \%$ 和 $100 \%$, 化合物 $10 \mathrm{a}, 10 \mathrm{~b}$ 和 $10 \mathbf{j}$ 对蚜虫的抑制率分别为 $100 \%, 90 \%$ 和 $50 \%$. 另外, 部分标题化合物还表现出一 定的抗肿瘤作用. 其中化合物 10b, 10c 和 10e 对 HepG2 细胞具有较好的抗肿瘤活性, 其 $\mathrm{IC}_{50}$ 值分别为 $14.2,18.9$ 和 $16.4 \mu \mathrm{mol} / \mathrm{L}$, 要高于或接近于对照药 Sorafenib 的数 值, 具有进一步研究的价值.

\section{3 实验部分}

\section{1 仪器与试剂}

X-4 型数字显示熔点测定仪(北京泰克仪器有限公 司), 温度计未经校正; Yanaco-CHN CORDER MT-3 自 动元素分析仪; Bruker AM-400 型核磁共振仪, 以 $d_{6}$-DMSO 或 $\mathrm{CDCl}_{3}$ 为溶剂, TMS 为内标; 柱层析硅胶为 $\mathrm{H}$ 型(青岛海洋化工厂，200３00 目). 所用试剂均为分 析纯. 3-取代基- $1 H$-吡唑-5-甲酸乙酯(1)和 1-甲基-3-取代 基吡唑-5-甲酸乙酯(2)参照文献[28]方法制备. 1-甲基-3取代基-4-氯吡唑-5-甲酸乙酯(3)、1-甲基-3-取代基-4-氯 吡唑-5-甲酸(4)和 1-甲基-3-取代基-4-氯吡唑-5-甲酰氯 (5)参照文献[29]方法制备. 5-取代苯基异噁唑-3-甲酸甲 酯(6)参照文献[30]方法制备. 化合物 7、8 和 9 参照文献 [21]方法制备.

\section{2 目标化合物 10 的合成}

在一 $50 \mathrm{~mL}$ 圆底烧瓶中, 加入 $4 \mathrm{mmol}$ 中间体 9、5 $\mathrm{mmol}$ 三乙胺及 $20 \mathrm{~mL} \mathrm{THF}$, 冰浴下搅拌, 向其中慢慢滴 加 $4 \mathrm{mmol} 1$-甲基-3-取代基-4-氯吡唑-5-甲酰氯(5)的 $\mathrm{THF}$ 溶液 $(5 \mathrm{~mL})$. 滴毕, 继续室温搅拌 $6 \sim 15 \mathrm{~h}$. 停止反 应, 减压蒸除溶剂, 向残余物中加入 $40 \mathrm{~mL}$ 二氯甲烷, 用水洗涤数次, 无水硫酸钠干燥, 抽滤, 母液脱溶, 所 得残余物经柱层析 $[V$ (石油醚 $): V($ 乙酸乙酯 $)=3: 1]$ 分 离得到目标化合物 $10 a \sim 10 p$.

1-甲基-3-乙基-4-氯- $N$ - $\{$ [5-(4-甲氧基苯基)异噁唑-3基]甲基\}-1H-吡唑-5-甲酰胺(10a)：白色固体，产率 62\%. m.p. $154 \sim 155{ }^{\circ} \mathrm{C} ;{ }^{1} \mathrm{H}$ NMR (400 MHz, $\left.\mathrm{CDCl}_{3}\right) \delta: 7.71$ (d, $J=8.8 \mathrm{~Hz}, 2 \mathrm{H}, \mathrm{ArH}), 7.33$ (s, 1H, NH), 6.97 (d, $J=8.8$ $\mathrm{Hz}, 2 \mathrm{H}, \mathrm{ArH}), 6.44$ (s, 1H, isoxazole-H), 4.74 (d, $J=5.6$ $\left.\mathrm{Hz}, 2 \mathrm{H}, \mathrm{CH}_{2}\right), 4.16\left(\mathrm{~s}, 3 \mathrm{H}, \mathrm{NCH}_{3}\right), 3.86$ (s, 3H, $\mathrm{ArOCH}_{3}$ ), $2.65\left(\mathrm{q}, J=7.6 \mathrm{~Hz}, 2 \mathrm{H}, \mathrm{CH}_{2}\right), 1.24(\mathrm{t}, J=7.6 \mathrm{~Hz}, 3 \mathrm{H}$, $\left.\mathrm{CH}_{3}\right) ;{ }^{13} \mathrm{C}$ NMR (100 MHz, $\left.\mathrm{CDCl}_{3}\right) \delta: 170.7,161.3,161.1$, $158.8,149.7,130.6,127.5,120.0,114.4,108.1,97.3,55.4$, 40.8, 35.6, 19.2, 12.8. Anal. calcd for $\mathrm{C}_{18} \mathrm{H}_{19} \mathrm{ClN}_{4} \mathrm{O}_{3}: \mathrm{C}$ 57.68, H 5.11, N 14.95; found C 57.57, H 5.21, N 14.83.

1-甲基-3-乙基-4-氯- $N$-\{[5-(4-甲基苯基)异噁唑-3基]甲基\}-1H-吡唑-5-甲酰胺(10b): 白色固体, 产率 $60 \%$. m.p. $152 \sim 153{ }^{\circ} \mathrm{C} ;{ }^{1} \mathrm{H}$ NMR (400 MHz, $d_{6}$-DMSO) $\delta: 9.00$ (s, 1H, NH), 7.76 (d, J=8.0 Hz, 2H, ArH), 7.35 (d, $J=8.0 \mathrm{~Hz}, 2 \mathrm{H}, \mathrm{ArH}), 6.88(\mathrm{~s}, 1 \mathrm{H}$, isoxazole-H), 4.57 (d, $\left.J=5.6 \mathrm{~Hz}, 2 \mathrm{H}, \mathrm{CH}_{2}\right), 3.89$ (s, 3H, $\left.\mathrm{NCH}_{3}\right), 2.57$ (q, $J=7.6$ $\left.\mathrm{Hz}, 2 \mathrm{H}, \mathrm{CH}_{2}\right), 2.37$ (s, 3H, $\left.\mathrm{ArCH}_{3}\right), 1.18$ (t, J=7.6 Hz, 3H, $\left.\mathrm{CH}_{3}\right) ;{ }^{13} \mathrm{C} \mathrm{NMR}\left(100 \mathrm{MHz}, \mathrm{CDCl}_{3}\right) \delta: 170.9,161.1,158.8$, 149.7, 140.8, 130.6, 129.7, 125.8, 124.4, 108.1, 98.1, 40.8, 35.6, 21.5, 19.2, 12.8. Anal. calcd for $\mathrm{C}_{18} \mathrm{H}_{19} \mathrm{ClN}_{4} \mathrm{O}_{2}$ : C 60.25, H 5.34, N 15.61; found C 60.12, H 5.46, N 15.70.

1- 甲基-3-乙基-4-氯- $N$-[(5- 苯基异噁唑-3-基) 甲 基]- $1 H$-吡唑-5-甲酰胺(10c): 白色固体，产率 56\%. m.p. $126 \sim 127{ }^{\circ} \mathrm{C}$; ${ }^{1} \mathrm{H}$ NMR (400 MHz, $d_{6}$-DMSO) $\delta$ : 9.02 (s, $1 \mathrm{H}, \mathrm{NH}), 7.86 \sim 7.89(\mathrm{~m}, 2 \mathrm{H}, \mathrm{ArH}), 7.49 \sim 7.57(\mathrm{~m}, 3 \mathrm{H}$, $\operatorname{ArH}), 6.96(\mathrm{~s}, 1 \mathrm{H}$, isoxazole-H), $4.58(\mathrm{~d}, J=5.6 \mathrm{~Hz}, 2 \mathrm{H}$, $\mathrm{CH}_{2}$ ), 3.89 (s, 3H, $\mathrm{NCH}_{3}$ ), 2.57 (q, J=7.6 Hz, 2H, $\mathrm{CH}_{2}$ ), $1.18\left(\mathrm{t}, J=7.6 \mathrm{~Hz}, 3 \mathrm{H}, \mathrm{CH}_{3}\right) ;{ }^{13} \mathrm{C} \mathrm{NMR}\left(100 \mathrm{MHz}, \mathrm{CDCl}_{3}\right)$ $\delta: 170.8,161.2,158.8,149.7,130.6,130.4,129.0,127.1$, 125.9, 108.1, 98.7, 40.8, 35.5, 19.2, 12.8. Anal. calcd for $\mathrm{C}_{17} \mathrm{H}_{17} \mathrm{ClN}_{4} \mathrm{O}_{2}$ : C 59.22, $\mathrm{H} 4.97, \mathrm{~N} \mathrm{16.25}$; found $\mathrm{C} 59.35$, $\mathrm{H} 4.85$, N 16.16.

1-甲基-3-乙基-4-氯- $N$-\{[5-(3-氟苯基)异噁唑-3-基] 甲基 $\}-1 H$-吡唑-5-甲酰胺(10d): 白色固体，产率 50\%. m.p. $150 \sim 151{ }^{\circ} \mathrm{C} ;{ }^{1} \mathrm{H}$ NMR $\left(100 \mathrm{MHz}, \mathrm{CDCl}_{3}\right) \delta: 7.56$ (d, $J=8.0 \mathrm{~Hz}, 1 \mathrm{H}, \mathrm{ArH}), 7.42 \sim 7.49(\mathrm{~m}, 2 \mathrm{H}, \mathrm{ArH}), 7.37$ $(\mathrm{s}, 1 \mathrm{H}, \mathrm{NH}), 7.13 \sim 7.17(\mathrm{~m}, 1 \mathrm{H}, \mathrm{ArH}), 6.61(\mathrm{~s}, 1 \mathrm{H}$, isoxazole-H), $4.77\left(\mathrm{~d}, J=5.6 \mathrm{~Hz}, 2 \mathrm{H}, \mathrm{CH}_{2}\right), 4.17(\mathrm{~s}, 3 \mathrm{H}$, $\left.\mathrm{NCH}_{3}\right), 2.66\left(\mathrm{q}, J=7.6 \mathrm{~Hz}, 2 \mathrm{H}, \mathrm{CH}_{2}\right), 1.25$ (t, $J=7.6 \mathrm{~Hz}$, $\left.3 \mathrm{H}, \mathrm{CH}_{3}\right) ;{ }^{13} \mathrm{C} \mathrm{NMR}\left(100 \mathrm{MHz}, \mathrm{CDCl}_{3}\right) \delta: 169.4,164.2$, $161.7,161.4,158.8,149.7,130.8,129.0,121.6,117.5$, 113.0, 108.1, 99.6, 40.8, 35.5, 19.2, 12.8. Anal. calcd for $\mathrm{C}_{17} \mathrm{H}_{16} \mathrm{ClFN}_{4} \mathrm{O}_{2}$ : C 56.28, H 4.45, N 15.44; found C 56.16, $\mathrm{H} 4.51$, N 15.33 .

1-甲基-3-乙基-4-氯- $N$ - $\{$ [5-(4-氟苯基)异噁唑-3-基 $]$ 甲基 $\}-1 H$-吡唑-5-甲酰胺(10e)：白色固体，产率 54\%. m.p. $146 \sim 148{ }^{\circ} \mathrm{C} ;{ }^{1} \mathrm{H}$ NMR $\left(100 \mathrm{MHz}, \mathrm{CDCl}_{3}\right) \delta: 7.77 \sim$ $7.80(\mathrm{~m}, 2 \mathrm{H}, \mathrm{ArH}), 7.35(\mathrm{~s}, 1 \mathrm{H}, \mathrm{NH}), 7.16 \sim 7.20(\mathrm{~m}, 2 \mathrm{H}$, ArH), $6.54(\mathrm{~s}, 1 \mathrm{H}$, isoxazole-H), $4.77(\mathrm{~d}, J=8.0 \mathrm{~Hz}, 2 \mathrm{H}$, $\mathrm{CH}_{2}$ ), 4.18 (s, 3H, $\left.\mathrm{NCH}_{3}\right), 2.67$ (q, $J=8.0 \mathrm{~Hz}, 2 \mathrm{H}, \mathrm{CH}_{2}$ ), $1.26\left(\mathrm{t}, J=8.0 \mathrm{~Hz}, 3 \mathrm{H}, \mathrm{CH}_{3}\right) ;{ }^{13} \mathrm{C} \mathrm{NMR}\left(100 \mathrm{MHz}, \mathrm{CDCl}_{3}\right)$ $\delta: 169.8,165.1,162.6,161.3,158.8,149.7,130.5,128.0$, 127.9, 123.5, 116.4, 108.1, 98.6, 40.8, 35.5, 19.2, 12.8. Anal. calcd for $\mathrm{C}_{17} \mathrm{H}_{16} \mathrm{ClFN}_{4} \mathrm{O}_{2}$ : C 56.28, H 4.45, N 15.44; found $\mathrm{C}$ 56.21, $\mathrm{H} 4.36, \mathrm{~N} 15.56$.

1-甲基-3-乙基-4-氯- $N$ - $\{$ [5-(4-氯苯基)异啞唑-3-基] 甲基\}-1H-吡唑-5-甲酰胺(10f): 白色固体，产率 58\%. m.p. $188 \sim 189{ }^{\circ} \mathrm{C} ;{ }^{1} \mathrm{H}$ NMR (400 MHz, $d_{6}$-DMSO) $\delta$ : 
9.01 (s, 1H, NH), 7.90 (d, $J=8.4 \mathrm{~Hz}, 2 \mathrm{H}, \operatorname{ArH}), 7.61$ (d, $J=8.8 \mathrm{~Hz}, 2 \mathrm{H}, \mathrm{ArH}), 7.01(\mathrm{~s}, 1 \mathrm{H}$, isoxazole-H), $4.58(\mathrm{~d}$, $\left.J=5.6 \mathrm{~Hz}, 2 \mathrm{H}, \mathrm{CH}_{2}\right), 3.89\left(\mathrm{~s}, 3 \mathrm{H}, \mathrm{NCH}_{3}\right), 2.57$ (q, $J=7.6$ $\left.\mathrm{Hz}, 2 \mathrm{H}, \mathrm{CH}_{2}\right), 1.17\left(\mathrm{t}, J=7.6 \mathrm{~Hz}, 3 \mathrm{H}, \mathrm{CH}_{3}\right) ;{ }^{13} \mathrm{C} \mathrm{NMR}$ $\left(100 \mathrm{MHz}, \mathrm{CDCl}_{3}\right) \delta: 169.6,161.4,158.8,149.7,136.5$, 130.5, 129.4, 127.1, 125.6, 108.1, 99.1, 40.8, 35.5, 19.2, 12.8. Anal. calcd for $\mathrm{C}_{17} \mathrm{H}_{16} \mathrm{Cl}_{2} \mathrm{~N}_{4} \mathrm{O}_{2}$ : C 53.84, $\mathrm{H} 4.25, \mathrm{~N}$ 14.77; found C 53.96, H 4.15, N 14.68 .

1-甲基-3-乙基-4-氯- $N$ - $\{$ [5-(3-溴苯基)异噁唑-3-基] 甲基\}-1H-吡唑-5-甲酰胺(10g): 白色固体, 产率 52\%. m.p. $130 \sim 131{ }^{\circ} \mathrm{C} ;{ }^{1} \mathrm{H}$ NMR $\left(100 \mathrm{MHz}, \mathrm{CDCl}_{3}\right) \delta: 7.34 \sim$ $7.93(\mathrm{~m}, 5 \mathrm{H}, \mathrm{ArH}$ and $\mathrm{NH}), 6.61$ (s, $1 \mathrm{H}$, isoxazole-H), 4.77 (d, $J=5.6 \mathrm{~Hz}, 2 \mathrm{H}, \mathrm{CH}_{2}$ ), 4.18 (s, 3H, $\mathrm{NCH}_{3}$ ), 2.67 (q, $J=$ $7.6 \mathrm{~Hz}, 2 \mathrm{H}, \mathrm{CH}_{2}$ ), 1.26 (t, $\left.J=7.6 \mathrm{~Hz}, 3 \mathrm{H}, \mathrm{CH}_{3}\right) ;{ }^{13} \mathrm{C} \mathrm{NMR}$ $\left(100 \mathrm{MHz}, \mathrm{CDCl}_{3}\right) \delta: 169.1,161.4,158.8,149.7,133.3$, 130.6, 129.0, 128.8, 125.9, 124.4, 123.1, 108.1, 99.6, 40.8, 35.4, 19.2, 12.8. Anal. calcd for $\mathrm{C}_{17} \mathrm{H}_{16} \mathrm{BrClN}_{4} \mathrm{O}_{2}$ : C 48.19, H 3.81, N 13.22; found C 48.06, H 3.93, N 13.32 .

1-甲基-3-乙基-4-氯- $N$ - $\{[5$-(3,4-二甲氧基苯基)异噁 唑-3-基]甲基\}-1 $H$-吡唑-5-甲酰胺(10h): 白色固体, 产率 55\%. m.p. $155 \sim 157{ }^{\circ} \mathrm{C} ;{ }^{1} \mathrm{H}$ NMR $\left(100 \mathrm{MHz}, \mathrm{CDCl}_{3}\right) \delta$ : $7.35 \sim 7.37(\mathrm{~m}, 2 \mathrm{H}, \mathrm{ArH}), 6.94(\mathrm{~d}, J=8.4 \mathrm{~Hz}, 1 \mathrm{H}, \operatorname{ArH})$, $6.48(\mathrm{~s}, 1 \mathrm{H}$, isoxazole- $\mathrm{H}), 4.75\left(\mathrm{~d}, J=5.6 \mathrm{~Hz}, 2 \mathrm{H}, \mathrm{CH}_{2}\right)$, $4.18\left(\mathrm{~s}, 3 \mathrm{H}, \mathrm{NCH}_{3}\right), 3.96\left(\mathrm{~s}, 3 \mathrm{H}, \mathrm{ArOCH}_{3}\right), 3.95(\mathrm{~s}, 3 \mathrm{H}$, $\left.\mathrm{ArOCH}_{3}\right), 2.66\left(\mathrm{q}, J=7.6 \mathrm{~Hz}, 2 \mathrm{H}, \mathrm{CH}_{2}\right), 1.25$ (t, $J=7.6$ $\left.\mathrm{Hz}, 3 \mathrm{H}, \mathrm{CH}_{3}\right) ;{ }^{13} \mathrm{C}$ NMR $\left(100 \mathrm{MHz}, \mathrm{CDCl}_{3}\right) \delta: 170.7$, $161.2,158.8,150.9,149.7,149.3,130.6,120.1,119.2$, 111.2, 108.6, 108.1, 97.6, 56.1, 56.0, 40.8, 35.5, 19.2, 12.8 . Anal. calcd for $\mathrm{C}_{19} \mathrm{H}_{21} \mathrm{ClN}_{4} \mathrm{O}_{4}$ : C 56.37, H 5.23, N 13.84; found $\mathrm{C} 56.26, \mathrm{H} 5.35, \mathrm{~N} 13.72$.

1-甲基-3-乙基-4-氯- $N$ - $\{[5$-(2,4-二氟苯基)异噁唑-3基]甲基\}-1H-吡唑-5-甲酰胺(10i)：白色固体，产率 48\%. m.p. $137 \sim 139{ }^{\circ} \mathrm{C} ;{ }^{1} \mathrm{H}$ NMR $\left(100 \mathrm{MHz}, \mathrm{CDCl}_{3}\right) \delta: 7.94 \sim$ 8.00 (m, 1H, ArH), 7.35 (s, 1H, NH), 6.96 7.08 (m, 2H, $\mathrm{ArH}), 6.72(\mathrm{~s}, 1 \mathrm{H}$, isoxazole-H), $4.80(\mathrm{~d}, J=5.6 \mathrm{~Hz}, 2 \mathrm{H}$, $\mathrm{CH}_{2}$ ), 4.18 (s, $3 \mathrm{H}, \mathrm{NCH}_{3}$ ), 2.67 (q, $J=7.6 \mathrm{~Hz}, 2 \mathrm{H}, \mathrm{CH}_{2}$ ), $1.26\left(\mathrm{t}, J=7.6 \mathrm{~Hz}, 3 \mathrm{H}, \mathrm{CH}_{3}\right) ;{ }^{13} \mathrm{C} \mathrm{NMR}\left(100 \mathrm{MHz}, \mathrm{CDCl}_{3}\right)$ $\delta: 169.6,163.8,162.8,161.5,158.7,149.7,136.0,130.5$, 128.9, 112.3, 108.1, 104.9, 102.2, 40.8, 35.5, 19.2, 12.8 . Anal. calcd for $\mathrm{C}_{17} \mathrm{H}_{15} \mathrm{ClF}_{2} \mathrm{~N}_{4} \mathrm{O}_{2}$ : C 53.62, H 3.97, N 14.71; found $\mathrm{C} 53.70, \mathrm{H} 3.85, \mathrm{~N} 14.82$.

1-甲基-3-乙基-4-氯- $N$ - $\{[5$-(3,4-二氯苯基)异噁唑-3基]甲基\}-1 $H$-吡唑-5-甲酰胺(10j): 白色固体, 产率 51\%. m.p. $148 \sim 150{ }^{\circ} \mathrm{C} ;{ }^{1} \mathrm{H}$ NMR $\left(100 \mathrm{MHz}, \mathrm{CDCl}_{3}\right) \delta: 7.88$ (d, $J=2.0 \mathrm{~Hz}, 1 \mathrm{H}, \mathrm{ArH}$ ), $7.56 \sim 7.64$ (m, 2H, ArH), 7.35 (s, $1 \mathrm{H}, \mathrm{NH}), 6.62(\mathrm{~s}, 1 \mathrm{H}$, isoxazole-H), $4.77(\mathrm{~d}, J=5.6 \mathrm{~Hz}$, $\left.2 \mathrm{H}, \mathrm{CH}_{2}\right), 4.18\left(\mathrm{~s}, 3 \mathrm{H}, \mathrm{NCH}_{3}\right), 2.67(\mathrm{q}, J=7.6 \mathrm{~Hz}, 2 \mathrm{H}$, $\left.\mathrm{CH}_{2}\right), 1.26\left(\mathrm{t}, J=7.6 \mathrm{~Hz}, 3 \mathrm{H}, \mathrm{CH}_{3}\right) ;{ }^{13} \mathrm{C}$ NMR $(100 \mathrm{MHz}$, $\left.\mathrm{CDCl}_{3}\right) \delta: 168.3,161.5,158.8,149.7,134.7,133.6,131.2$, 130.4, 127.6, 126.9, 124.9, 108.1, 99.9, 40.8, 35.4, 19.2, 12.8. Anal. calcd for $\mathrm{C}_{17} \mathrm{H}_{15} \mathrm{Cl}_{3} \mathrm{~N}_{4} \mathrm{O}_{2}$ : C 49.36, H 3.65, N 13.54; found C 49.27, H 3.73, N 13.65 .

1-甲基-3-(4-甲氧基苯基)-4-氯- $N$ - $\{$ [5-(4-氟苯基)异 噁唑-3-基]甲基 $\}$ - $1 H$-吡唑-5-甲酰胺(10k): 白色固体, 产 率 53\%. m.p. 226 228 ${ }^{\circ} \mathrm{C} ;{ }^{1} \mathrm{H}$ NMR $\left(100 \mathrm{MHz}, \mathrm{CDCl}_{3}\right)$ $\delta: 7.87$ (d, $J=4.0 \mathrm{~Hz}, 1 \mathrm{H}, \mathrm{ArH}), 7.70 \sim 7.79(\mathrm{~m}, 4 \mathrm{H}, \mathrm{ArH})$, 7.40 (s, 1H, ArH), 7.14 7.19 (m, 2H, ArH), 7.00 (d, $J=$ $8.0 \mathrm{~Hz}, 1 \mathrm{H}, \mathrm{ArH}), 6.54(\mathrm{~s}, 1 \mathrm{H}$, isoxazole-H), $4.79(\mathrm{~d}, J=$ $\left.4.0 \mathrm{~Hz}, 2 \mathrm{H}, \mathrm{CH}_{2}\right), 4.25\left(\mathrm{~s}, 3 \mathrm{H}, \mathrm{NCH}_{3}\right), 3.95(\mathrm{~s}, 3 \mathrm{H}$, $\left.\mathrm{ArOCH}_{3}\right) ;{ }^{13} \mathrm{C} \mathrm{NMR}\left(100 \mathrm{MHz}, \mathrm{CDCl}_{3}\right) \delta: 169.9,165.1$, $162.6,161.2,158.6,155.2,145.0,131.8,129.3,128.9$, $128.0,127.0,124.2,122.7,116.2,114.0,111.9,107.1$, 98.5, 56.2, 41.3, 35.7. Anal. calcd for $\mathrm{C}_{22} \mathrm{H}_{18} \mathrm{ClFN}_{4} \mathrm{O}_{3}$ : C 59.94, H 4.12, N 12.71; found C 59.82, H 4.01, N 12.62.

1-甲基-3-(3-氟苯基)-4-氯- $N$ - \{[5-(4-氯苯基)异噁唑3-基]甲基\}-1 $H$-吡唑-5-甲酰胺(101)：白色固体，产率 46\%. m.p. $175 \sim 177{ }^{\circ} \mathrm{C} ;{ }^{1} \mathrm{H}$ NMR (100 MHz, $\left.\mathrm{CDCl}_{3}\right) \delta$ : $7.71(\mathrm{~d}, J=8.4 \mathrm{~Hz}, 2 \mathrm{H}, \mathrm{ArH}), 7.64(\mathrm{~d}, J=7.6 \mathrm{~Hz}, 1 \mathrm{H}$, $\operatorname{ArH}), 7.56(\mathrm{~d}, J=8.4 \mathrm{~Hz}, 1 \mathrm{H}, \mathrm{ArH}), 7.39 \sim 7.46(\mathrm{~m}, 4 \mathrm{H}$, ArH and NH), 7.07 7.11 (m, 1H, ArH), $6.58(\mathrm{~s}, 1 \mathrm{H}$, isoxazole-H), $4.79\left(\mathrm{~d}, J=5.6 \mathrm{~Hz}, 2 \mathrm{H}, \mathrm{CH}_{2}\right), 4.26(\mathrm{~s}, 3 \mathrm{H}$, $\left.\mathrm{NCH}_{3}\right) ;{ }^{13} \mathrm{C}$ NMR $\left(100 \mathrm{MHz}, \mathrm{CDCl}_{3}\right) \delta: 169.7,164.0$, $161.6,161.2,158.6,145.1,136.6,132.9,132.0,130.2$, $130.1,129.4,127.1,125.5,123.1,115.6,114.6,107.5$, 99.1, 41.4, 35.6. Anal. calcd for $\mathrm{C}_{21} \mathrm{H}_{15} \mathrm{Cl}_{2} \mathrm{FN}_{4} \mathrm{O}_{2}$ : C 56.64, H 3.40, N 12.58; found C 56.75, H 3.52, N 12.47 .

1-甲基-3-(4-氟苯基)-4-氯- $N$ - $\{$ [5-(4-氯苯基)异噁唑3-基]甲基 \}-1 $H$-吡唑-5-甲酰胺(10m)：白色固体，产率 48\%. m.p. $>280{ }^{\circ} \mathrm{C} ;{ }^{1} \mathrm{H}$ NMR $\left(100 \mathrm{MHz}, \mathrm{CDCl}_{3}\right) \delta$ : $7.79 \sim 7.82(\mathrm{~m}, 2 \mathrm{H}, \mathrm{ArH}), 7.72(\mathrm{~d}, J=8.4 \mathrm{~Hz}, 2 \mathrm{H}, \mathrm{ArH})$, $7.41 \sim 7.47(\mathrm{~m}, 3 \mathrm{H}, \mathrm{ArH}$ and $\mathrm{NH}), 7.12 \sim 7.16(\mathrm{~m}, 2 \mathrm{H}$, $\mathrm{ArH}), 6.59(\mathrm{~s}, 1 \mathrm{H}$, isoxazole-H), $4.79(\mathrm{~d}, J=5.6 \mathrm{~Hz}, 2 \mathrm{H}$, $\left.\mathrm{CH}_{2}\right), 4.25$ (s, $\left.3 \mathrm{H}, \mathrm{NCH}_{3}\right) ;{ }^{13} \mathrm{C} \mathrm{NMR}\left(100 \mathrm{MHz}, \mathrm{CDCl}_{3}\right) \delta$ : 169.7, 165.0, 161.2, 158.6, 157.3, 145.6, 140.7, 136.6, $131.8,129.5,129.4,127.1,126.9,125.6,115.7,99.1,41.3$, 35.6. Anal. calcd for $\mathrm{C}_{21} \mathrm{H}_{15} \mathrm{Cl}_{2} \mathrm{FN}_{4} \mathrm{O}_{2}$ : C 56.64, $\mathrm{H} \mathrm{3.40,} \mathrm{N}$ 12.58; found C 56.53, H 3.48, N 12.70 .

1-甲基-3-(4-氯苯基)-4-氯- $N$-\{ $\{5$-(4-氯苯基)异噁唑3-基]甲基 \}-1 $\mathrm{H}$-吡唑-5-甲酰胺(10n): 白色固体, 产率 $50 \%$. m.p. $219 \sim 220{ }^{\circ} \mathrm{C} ;{ }^{1} \mathrm{H}$ NMR (100 MHz, $\left.\mathrm{CDCl}_{3}\right) \delta$ : 
$7.78(\mathrm{~d}, J=8.4 \mathrm{~Hz}, 2 \mathrm{H}, \operatorname{ArH}), 7.71(\mathrm{~d}, J=8.8 \mathrm{~Hz}, 2 \mathrm{H}$, ArH), $7.41 \sim 7.46(\mathrm{~m}, 5 \mathrm{H}, \mathrm{ArH}$ and $\mathrm{NH}), 6.58(\mathrm{~s}, 1 \mathrm{H}$, isoxazole-H), $4.79\left(\mathrm{~d}, J=5.6 \mathrm{~Hz}, 2 \mathrm{H}, \mathrm{CH}_{2}\right), 4.25(\mathrm{~s}, 3 \mathrm{H}$, $\left.\mathrm{NCH}_{3}\right) ;{ }^{13} \mathrm{C} \mathrm{NMR}\left(100 \mathrm{MHz}, \mathrm{CDCl}_{3}\right) \delta: 169.7,161.2$, $159.1,158.6,136.6,134.6,129.4,129.3,128.8,127.1$, $125.5,115.7,107.4,104.1,99.1,41.4,35.6$. Anal. calcd for $\mathrm{C}_{21} \mathrm{H}_{15} \mathrm{Cl}_{3} \mathrm{~N}_{4} \mathrm{O}_{2}$ : C 54.63, H 3.27, N 12.13; found C 54.75, H 3.16, N 12.05 .

1-甲基-3-(4-碘苯基)-4-氯- $N$ - $\{[5$-(4-氯苯基)异啞唑3-基]甲基 $\}-1 H$-吡唑-5-甲酰胺(100): 白色固体, 产率 53\%. m.p. 190 $192{ }^{\circ} \mathrm{C}$; ${ }^{1} \mathrm{H}$ NMR $\left(100 \mathrm{MHz}, \mathrm{CDCl}_{3}\right) \delta$ : $7.71(\mathrm{~d}, J=8.4 \mathrm{~Hz}, 2 \mathrm{H}, \operatorname{ArH}), 7.65$ (d, $J=8.4 \mathrm{~Hz}, 2 \mathrm{H}$, $\operatorname{ArH}), 7.51(\mathrm{~d}, J=8.4 \mathrm{~Hz}, 2 \mathrm{H}, \operatorname{ArH}), 7.32 \sim 7.39(\mathrm{~m}, 3 \mathrm{H}$, ArH and $\mathrm{NH}), 6.51$ (s, 1H, isoxazole-H), 4.72 (d, $J=6.0$ $\left.\mathrm{Hz}, 2 \mathrm{H}, \mathrm{CH}_{2}\right), 4.18\left(\mathrm{~s}, 3 \mathrm{H}, \mathrm{NCH}_{3}\right) ;{ }^{13} \mathrm{C} \mathrm{NMR}(100 \mathrm{MHz}$, $\left.\mathrm{CDCl}_{3}\right) \delta: 168.7,160.1,157.5,144.3,136.7,135.5,130.9$, $129.9,129.3,128.4,128.2,126.1,124.5,106.4,98.0,93.6$, 40.4, 34.6. Anal. calcd for $\mathrm{C}_{21} \mathrm{H}_{15} \mathrm{Cl}_{2} \mathrm{IN}_{4} \mathrm{O}_{2}: \mathrm{C} 45.60, \mathrm{H}$ 2.73, N 10.13; found C 45.72, H 2.63, N 10.21.

1-甲基-3-(2,4-二氟苯基)-4-氯- $N$ - $\{[5$-(4-氟苯基)异 啞唑-3-基]甲基\}-1H-吡唑-5-甲酰胺(10p)：白色固体，产 率 43\%. m.p. 173 $174{ }^{\circ} \mathrm{C}$; ${ }^{1} \mathrm{H}$ NMR (100 $\mathrm{MHz}, \mathrm{CDCl}_{3}$ ) $\delta: 7.71(\mathrm{~d}, J=8.8 \mathrm{~Hz}, 2 \mathrm{H}, \mathrm{ArH}), 7.41 \sim 7.51$ (m, 4H, ArH and $\mathrm{NH}), 6.91 \sim 7.00(\mathrm{~m}, 2 \mathrm{H}, \operatorname{ArH}), 6.58(\mathrm{~s}, 1 \mathrm{H}$, isoxazole-H), $4.78\left(\mathrm{~d}, J=5.6 \mathrm{~Hz}, 2 \mathrm{H}, \mathrm{CH}_{2}\right), 4.28(\mathrm{~s}, 3 \mathrm{H}$, $\left.\mathrm{NCH}_{3}\right) ;{ }^{13} \mathrm{C}$ NMR (100 MHz, $\left.\mathrm{CDCl}_{3}\right) \delta: 169.7,161.2$, $158.5,142.4,136.6,132.4,132.3,131.4,129.4,127.1$, $125.6,114.7,111.7,111.5,109.5,104.5,99.1,41.5,35.6$. Anal. calcd for $\mathrm{C}_{21} \mathrm{H}_{14} \mathrm{ClF}_{3} \mathrm{~N}_{4} \mathrm{O}_{2}$ : C 56.45, H 3.16, N 12.54; found C 56.36, H 3.28, N 12.63.

\section{4 生物活性测试}

用分析天平称取一定质量的原药, 用含吐温 -80 乳化剂的 $N, N$-二甲基甲酰胺(DMF)溶解配制成 $1.0 \%$ 母 液, 然后用蒸馏水稀释备用. 每个处理 3 次重复, 设空 白对照.

杀虫活性测试所选昆虫分别为粘虫 (Oriental armyworm) 、蚜虫 (Aphis medicaginis) 、褐飞闽 (Nilaparvata lugens)和朱砂叶螨(Tetranychus cinnabari$n u s$ ). 对照药剂分别为吡螨胺(Tebufenpyrad)和吡虫啉 (Imidacloprid).

蚜虫、螨虫和褐飞亲: 采用喷雾法. 首先, 分别将接 有蚜虫、朱砂叶螨的蚕豆叶片和接有褐飞虫的水稻苗于 Potter 喷雾塔下喷雾处理, 处理后蚜虫置于 $20 \sim 22{ }^{\circ} \mathrm{C}$ 观察室内培养, 朱砂叶螨和褐飞虫置于 $24 \sim 27{ }^{\circ} \mathrm{C}$ 观察 室内培养, $2 \mathrm{~d}$ 后观察结果, 检查死活虫数, 并进行统计
分析. 粘虫: 采用浸叶碟法. 首先, 将适量玉米叶在配 好的药液中充分浸润后自然阴干，放入垫有滤纸的培养 III.中，接粘虫 3 龄中期幼虫 10 头/孟，置于 $24 \sim 27{ }^{\circ} \mathrm{C}$ 观 察室内培养, 调查药后 $2 \mathrm{~d}$ 的死活虫数, 并进行统计分 析.

抗肿瘤活性测试所用细胞株分别为人胃癌 (SGC7901)和人肝癌(HepG2)细胞株. 阳性对照药为索 拉菲尼(Sorafenib). 采用噻唑蓝(MTT)法测定了目标化 合物的体外抗肿瘤活性, 具体方法参照文献[31].

辅助材料(Supporting Information) 化合物 $10 \mathrm{a} \sim 10 \mathrm{p}$ 的 ${ }^{1} \mathrm{H}$ NMR 和 ${ }^{13} \mathrm{C}$ NMR 图谱. 这些材料可以免费从本刊 网站(http://sioc-journal.cn/)上下载.

\section{Referenes}

[1] Dai, H.; Li, Y. Q.; Du, D.; Qin, X.; Zhang, X.; Yu, H. B.; Fang, J. X. J. Agric. Food Chem. 2008, 56, 10805.

[2] Sun, Y. F.; Li. Y. Q.; Ling, Y.; Yu, H. L.; Yang, S. X.; Yang, X. L. Chin. J. Org. Chem. 2011, 31, 1425 (in Chinese). (孙玉凤, 李永强, 凌云, 宇红莲, 杨绍祥, 杨新玲, 有机化学, 2011, 31, 1425.)

[3] Wang, B. L.; Zhu, H. W.; Ma, Y.; Xiong, L. X.; Li, Y. Q.; Zhao, Y.; Zhang, J. F.; Chen, Y. W. Zhou, S.; Li, Z. M. J. Agric. Food Chem. 2013, 61,3 .

[4] Wu, Z. B.; Zhou, X.; Ye, Y. Q.; Wang, P. Y.; Yang, S. Chin. Chem. Lett. 2017, 28, 121.

[5] Li, Y.; Zhang, H. Q.; Liu, J.; Yang, X. P.; Liu, Z. J. J. Agric. Food Chem. 2006, 54, 3636.

[6] Zhang, J. F.; Liu, C.; Ma, Y.; Wang, B. L.; Xiong, L. X.; Yu, S. J.; Li, Z. M. Lett. Drug Des. Discovery 2013, 10, 497.

[7] Ouyang, G. P.; Cai, X. J.; Chen, Z.; Song, B. A.; Bhadury, P. S.; Yang, S.; Jin, L. H.; Xue, W.; Hu, D. Y.; Zeng, S. J. Agric. Food Chem. 2008, 56, 10160.

[8] Park, H. J.; Lee, K.; Park, S. J.; Ahn, B.; Lee, J. C.; Cho, H. Y.; Lee, K. I. Bioorg. Med. Chem. Lett. 2005, 15, 3307.

[9] Shi, Y. J.; Li, Y.; Fang, Y.; Ye, L. Y.; Chen, J.; Dai, H. Chin. J. Org Chem. 2016, 36, 1431 (in Chinese).

(石玉军, 李阳, 方源, 叶林玉，陈佳，戴红，有机化学, 2016, 36, 1431.)

[10] Gu, B. Q.; Zhu, W. Q.; Fan, W. Z.; Qian, H.; Liu, J. M.; Zhang, A. Q.; Shen, R. X. Modern Agrochem. 2002, 1, 9 (in Chinese). (顾保权, 朱伟清, 范文政, 钱虹, 刘建梅, 张爱庆, 沈荣仙, 现 代农药, 2002, 1, 9.)

[11] Lahm, G. P., Selby, T. P.; Freudenberger, J. H.; Stevenson, T. M.; Myers, B. J.; Seburyamo, G.; Smith, B. K; Flexner, L.; Clark, C. E.; Cordova, D. Bioorg. Med. Chem. Lett. 2005, 15, 4898.

[12] Penning, T. D.; Talley, J. J.; Bertenshaw, S. R.; Carter, J. S.; Collins, P. W.; Docter, S.; Graneto, M. J.; Lee, L. F.; Malecha, J. W.; Miyashiro, J. M.; Rogers, R. S.; Rogier, D. J.; Yu, S. S.; Anderson, G. D.; Burton, E. G.; Cogburn, J. N.; Gregory, S. A.; Koboldt, C. M.; Perkins, W. E. Seibert, K.; Veenhuizen, A. W.; Zhang, Y. Y.; Isakson, P. C. J. Med. Chem. 1997, 40, 1347.

[13] Teng, M.; Zhu, J. J.; Johnson, M. D.; Chen, P.; Kornmann, J.; Chen, E. T.; Blasina, A.; Register, J.; Anderes, K.; Rogers, C.; Deng, Y. L.; Ninkovic, S.; Grant, S.; Hu, Q. Y.; Lundgren, K.; Peng, Z. W.; Kania, R. S. J. Med. Chem. 2007, 50, 5253.

[14] Xu, S. S.; Zhang, M.; Zhang, L.; Jiang, H. F.; Zhu, N.; Song, L. P.; Deng, H. M. Chin. J. Org. Chem. 2015, 35, 2595 (in Chinese). (徐姗姗, 张敏, 张丽, 蒋海芳, 朱宁, 宋力平, 邓红梅, 有机化 学, 2015, 35, 2595.)

[15] Liu, T. T.; Ni, Y.; Zhong, L. K.; Huang, H. Y.; Hu. W. Q.; Xu. T. M.; 
Tan, C. X. Chin. J. Org. Chem. 2015, 35, 422 (in Chinese). (刘婷婷, 倪芸, 钟良坤, 黄红英, 胡伟群, 许天明, 谭成侠, 有 机化学, 2015, 35, 422.)

[16] Yong, J. P.; Lu, C. Z.; Wu, X. Y. Anti-Cancer Agents Med. Chem. $\mathbf{2 0 1 5}, 15,131$

[17] Kamal, A. K.; Reddy, J. S.; Ramaiah, M. J.; Dastagiri, D.; Bharathi, E. V.; Azhar, M. A.; Sultana, F.; Pushpavalli, S. N. C. V. L.; Bhadra, M. P. Juvekar, A.; Sen, S.; Zingde, S. Eur. J. Med. Chem. 2010, 45, 3924.

[18] Kamal, A. K.; Bharathi, E. V.; Reddy, J. S.; Ramaiah, M. J.; Dastagiri, D.; Reddy, M. K.; Viswanath, A.; Reddy, T. L.; Shaik, T. B.; Pushpavalli, S. N. C. V. L.; Bhadra, M. P. Eur. J. Med. Chem. 2011, 46, 691 .

[19] Stefan, A. L.; Simona, M.; Martina, D. F. Chem. Med. Chem. 2006, $1,197$.

[20] Lu, Q.; Shen, Q. Y.; Sun, L.; Wang, J. Y.; Song, G. H. Chin. J. Org. Chem. 2016, 36, 760 (in Chinese).

(陆青, 沈巧英, 孙璐, 王佳毅, 宋恭华, 有机化学, 2016, 36, 760.)

[21] Shi, Y. J.; Fang, Y.; Li, Y.; Chen, J.; Li, G.; Wang, Q. M.; Dai, H. Chem. J. Chin. Univ. 2017, 38, 421 (in Chinese). (石玉军, 方源, 李阳, 陈佳, 李刚, 汪清民, 戴红, 高等学校化 学学报, 2017, 38, 421.)

[22] Hu, D. J.; Liu, S. F.; Huang, T. H.; Tu, H. Y.; Zhang, A. D. Molecules 2009, 14, 1288.

[23] Dai, H.; Liu, J. B.; Miao, W. K.; Wu, S. S.; Zhang, X.; Wang, T. T.; Fang, J. X. Chin. J. Org. Chem. 2011, 31, 1943 (in Chinese).
(戴红, 刘建兵, 苗文科, 吴珊珊, 张欣, 王婷婷, 方建新, 有机 化学, 2011, 31, 1943.)

[24] Sheng, G. Z.; Zhang, W. Chin. J. Org. Chem. 2013, 33, 2271 (in Chinese).

(盛国柱，张炜，有机化学, 2013, 33, 2271.)

[25] Zhan, Y. Z.; Wang, B. L.; Zhang, L. Y.; Zhang, Y.; Zhang, X.; Li, Z. M.; Song, H. B. Acta Chim. Sinica. 2015, 73, 1173 (in Chinese) (詹益周, 王宝雷, 张丽媛, 张燕, 张晓, 李正名, 宋海斌, 化学 学报, 2015, 73, 1173.)

[26] Narita, K.; Matsuhara, K.; Itoh, J.; Akiyama, Y.; Dan, S.; Yamori, T.; Ito, A.; Yoshida, M.; Katoh, T. Eur. J. Med. Chem. 2016, 121, 592.

[27] Carta, D.; Bortolozzi, R.; Sturlese, M.; Salmaso, V.; Hamel, E.; Basso, G.; Calderan, L.; Quintieri, L.; Moro, S.; Viola, G.; Ferlin, M. G. Eur. J. Med. Chem. 2017, 127, 643.

[28] Wei, F.; Zhao, B. X.; Huang, B.; Zhang, L.; Sun, C. H.; Dong, W. L.; Shin, D. S.; Miao, J. Y. Bioorg. Med. Chem. Lett. 2006, 16, 6342.

[29] Song, H. J.; Liu, Y. X.; Xiong, L. X.; Li, Y. Q.; Yang, N.; Wang, Q. M. J. Agric. Food Chem. 2013, 61, 8730.

[30] Tanaka, A.; Terasawa, T.; Hagihara, H.; Sakuma, Y.; Ishibe, N.; Sawada, M.; Takasugi, H.; Tanaka, H. J. Med. Chem. 1998, 41, 2390.

[31] Liu, J. C.; Liu, Y. J.; He, H. W. Chin. J. Org. Chem. 2015, 35, 462 (in Chinese).

(刘建超, 刘勇军, 贺洪武, 有机化学, 2015, 35, 462.)

(Li, L.; Fan, Y.) 